\title{
El impacto de la Ley Fintech a la Industria de la Tecnología Financiera y el reto de establecer un Gobierno Corporativo
}

\author{
The impact of Fintech Law on the Financial Technology Industry and the challenge of \\ establishing a Corporate Governance
}

\begin{abstract}
Edith Urdanivia Gonzalez ${ }^{a}$, Adriana Zambrano Reyes ${ }^{b}$, Tomas Rodríguez Gómez $^{c}$
Abstract:

The growth of the Fintech industry created the need to establish a regulation for financial technology companies called Fintech Law, placing Mexico among the first countries to have such a regulation. But despite the benefits offered by this regulation to the Mexican financial system and the Fintech ecosystem itself, the growth of the financial technology industry could decrease due to such robust requirements, such as establishing a Corporate Government. That is why this document analyzes the impact of the Fintech Law on the ecosystem and how the growth of the financial technology industry can be affected in the short term.
\end{abstract}

Keywords:

Fintech, Fintech Ecosystem, Fintech Law, Corporate Governance

\section{Resumen:}

El crecimiento de la industria Fintech creo la necesidad de establecer una regulación para empresas tecnológicas financieras denominada Ley Fintech, posicionando a México entre los primeros países en poseer una regulación de este tipo. Pero a pesar de los beneficios que brinda esta regulación al sistema financiero mexicano y al propio ecosistema Fintech el crecimiento de la industria de la tecnología financiera podría disminuir debido a los requisitos tan robustos, como lo es el de establecer un Gobierno Corporativo. Es por ello que el presente documento analiza cual fue el impacto de la Ley Fintech al ecosistema y como el crecimiento de la industria tecnológico financiera puede verse afectado en el corto plazo.

\section{Palabras Clave:}

Fintech, Ecosistema Fintech, Ley Fintech, Gobierno Corporativo

\section{Introducción}

Los avances tecnológicos han llevado al mundo a la cuarta revolución industrial conocida como industria 4.0. Esta revolución viene dada por nuevas tecnologías como Ciencias de Datos, Inteligencia Artificial, Internet de las Cosas y Blockchain, las cuales han generado grandes oportunidades para la industria de la tecnología financiera mejor conocido como Fintech.

El termino Fintech que en español se traduce como Finanzas Tecnológicas, se originó desde hace al menos
150 años con la creación de la cinta Ticker, el medio electrónico más antiguo utilizado para la transmisión de los precios de las acciones a través del telégrafo. Sin embargo la industria Fintech ha dado un salto exponencial en los últimos años, debió a que las Startups se han enfocado en mejorar los servicios financieros para hacerlos más eficientes.

Las Starups Fintech buscan mejorar las condiciones ofrecidas por el sistema financiero tradicional a través del uso de la tecnología, de esta manera los servicios que ofrecen son menos costosos y más eficientes. Estas

\footnotetext{
a Estudiante de la Licenciatura en Ingeniería Económica y Financiera, Universidad Autónoma del Estado de Hidalgo, Instituto de Ciencias Económico Administrativas, https://orcid.org/0000-0001-8767-8401,Email: urdanivia.esap.30@gmail.com

b Profesora Investigadora de la Licenciatura en Ingeniería Económica y Financiera, Universidad Autónoma del Estado de Hidalgo, Instituto de Ciencias Económico Administrativas, https://orcid.org/0000-0002-9536-9403,Email: adriana_zambrano@uaeh.edu.mx
}

c Profesor Investigador de la Licenciatura en Ingeniería Económica y Financiera, Universidad Autónoma del Estado de Hidalgo, Instituto de Ciencias Económico Administrativas, https://orcid.org/0000-0001-8672-5018,Email: yhwh13@gmail.com 
empresas actúan como intermediarios en transacciones financieras, compra y venta de títulos financieros, así como asesoramiento financiero. Actualmente las Startups Fintech se están desarrollando en áreas como pagos y remesas, prestamos, gestión de finanzas personales y empresariales, crowdfunding, gestión de inversiones, seguros, trading y mercados, educación financiera y ahorro.

Hoy en día países de todo el mundo están comenzando a madurar su Ecosistema Fintech. Tal es el caso de México que logró posicionarse en 2019 como el primer Ecosistema Fintech de América Latina con 394 Startups, esto de acuerdo con la última edición disponible del informe titulado "Fintech en América Latina 2018: Crecimiento y Consolidación", publicado por el Banco Interamericano de Desarrollo (BID) y Finnovista. [1]

El increíble crecimiento del Ecosistema Fintech está obligando a los gobiernos de los países a regular la industria de la tecnología financiera. En México a través de la Secretaria de Hacienda y Crédito Público (SHCP), diferentes autoridades financieras y otros actores, trabajaron en el desarrollo de la Ley para Regular las Instituciones de Tecnología Financiera, denominada como "Ley Fintech". La Ley Fintech fue publicada el 9 de marzo de 2018 en el Diario Oficial de la Federación, colocando a México en tre los primeros países pioneros en el tema de regulación a Startups Fintech, denominadas por dicha ley como Institu ciones de Tecnología Financiera (ITF). Países como Holanda, Abu Dhabi, Canadá, Reino Unido, Malasia, Australia, Singapur y Hong Kong también cuentan con políticas regulatorias en marcha en el tema de Fintech. [2]

La Ley Fintech busca regular la organización, operación y funcionamiento de las Instituciones de Tecnología Financiera para brindar confianza y certeza a los usuarios de los servicios y productos que estas ofrecen, así como mitigar el riego de lavado de dinero y financiamiento al terrorismo.

Algunos de los requisitos para operar de acuerdo ha esta ley son el establecimiento de controles internos y de administración de riesgos, mecanismos de seguridad de la información y políticas de confidencialidad. La regulación también establece una vigilancia estricta de sanas prácticas societarias, de auditoria, de contabilidad, de revelación de informacion y de transparencia. En concreto uno de los principales requisitos que establece la Ley Fintech es la conformación de un Gobierno Corporativo. [3]

Un Gobierno corporativo es un conjunto prácticas y controles que se establecen con el objetivo de llevar una administración transparente y equitativa alineada con los intereses de sus stakeholders, teniendo como objeto prevenir conflictos de intereses y posibles abusos. Pero para la mayoría de las Startups Fintech el establecimiento de un Gobierno Corporativo resulta ser complicado y requiere de un tiempo considerable.

A pesar de los beneficios que brinda una regulación de este tipo al sistema financiero mexicano y al propio Ecosistema Fintech, el crecimiento de la industria de la tecnología financiera podría disminuir debido a los requisitos tan robustos, como lo es el de establecer un Gobierno Corporativo.

El pasado 25 de septiembre de 2019 fue la fecha límite para que las Startups Fintech entregaran su solicitud ante la Comisión Nacional Bancaria y de Valores (CNBV) para poder operar de forma legal, por lo que el presente trabajo analiza cual fue el impacto de la regulación al Ecosistema Fintech y como el crecimiento se la industria de la tecnología financiera puede verse disminuido en el corto plazo por la Ley Fintech.

\section{La Industria Tecnológico Financiera (Fintech) en México}

El Ecosistema Fintech a lo largo de la historia ha pasado por tres etapas evolutivas. En la primera etapa conocida como Fintech 1.0 los bancos comenzaron a utilizar computadoras, aparecieron los cajeros automáticos, las terminales puntos de venta para el pago con tarjeta de crédito y el trading electrónico. En la segunda etapa Fintech 2.0 comenzó el uso del internet, las páginas web y la banca digital. En la tercer y más reciente etapa denominada Fintech 3.0 comienza a desarrollarse el mundo de las startups basadas en el uso de tecnologías como Big Data, Machine Learning, Blockchain, Apps, etc. El ecosistema en el que se desarrollan actualmente las Fintech en México, es un "ecosistema heterogéneo e instrumental donde conviven emprendedores, gobiemo, fondos, aceleradoras e instituciones financieras tradicionales" (BANCOMEXT \& Finnovista, 2018) [4]. Este ecosistema está compuesto por seis elementos: Startups Fintech, desarrolladores de tecnología, Gobierno, clientes financieros, instituciones financieras tradicionales $y$ fondos de inversión y aceleradoras.

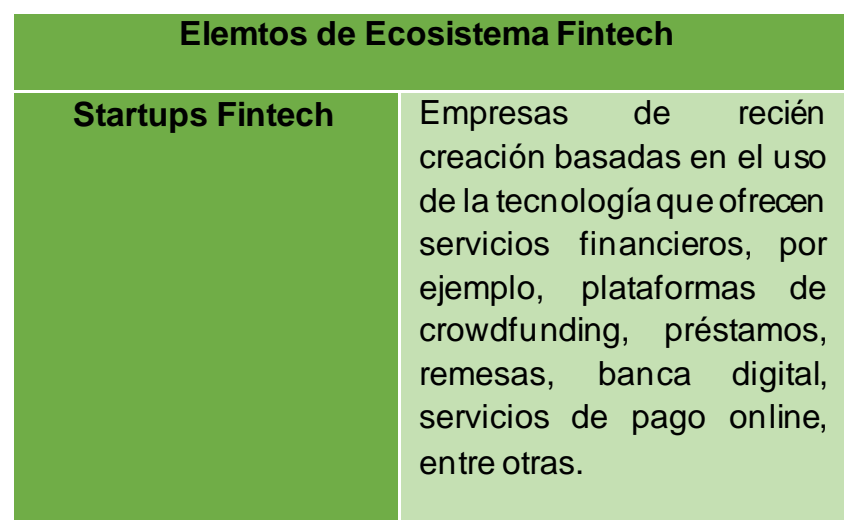




\begin{tabular}{|c|c|}
\hline $\begin{array}{l}\text { Desarrolladores de } \\
\text { tecnología }\end{array}$ & $\begin{array}{l}\text { Proveedores y creadores de } \\
\text { la tecnología que sustentan } \\
\text { a las Startups. }\end{array}$ \\
\hline Gobierno & $\begin{array}{l}\text { Agente que se encarga de } \\
\text { regular las operaciones de } \\
\text { las Fintech Startups. }\end{array}$ \\
\hline Clientes financieros & $\begin{array}{l}\text { Todos los individuos e } \\
\text { instituciones que hacen uso } \\
\text { de los servicios y productos } \\
\text { de las Startups Fintech. }\end{array}$ \\
\hline $\begin{array}{c}\text { Instituciones } \\
\text { financieras } \\
\text { tradicionales }\end{array}$ & $\begin{array}{l}\text { Instituciones bancarias, } \\
\text { compañías de seguros, } \\
\text { casas de bolsa, entre otras. }\end{array}$ \\
\hline $\begin{array}{l}\text { Fondos de inversión } \\
\text { y aceleradoras }\end{array}$ & $\begin{array}{l}\text { Instituciones que se } \\
\text { encargan de impulsar el } \\
\text { desarrollo de las Startups } \\
\text { Fintech. }\end{array}$ \\
\hline
\end{tabular}

Tabla 1. Descripción del Ecosistema Fintech Fuente: Elaboración propia a partir de información de BANCOMEXT, Finnovista, "Fintech en el mundo. La revolución digital de las finanzas llego a México", 2018, p.16.

Las Startups Fintech son en su mayoría emprendimientos que abarcan nichos específicos de mercado, brindando servicios más personalizados y eficientes que los que ofrecen las instituciones financieras tradicionales. Su principal detonante de crecimiento ha sido su habilidad para desagregar los servicios financieros. Ahora los consumidores no necesitan asistir y depender de una única institución para realizar operaciones financieras.

Es gracias a los desarrolladores de tecnología que las Startups Fintech lanzan al mercado servicios financieros innovadores, a través de plataformas, análisis de Big Data, aplicaciones móviles, entre otras. Por ejemplo, las tecnologías de Big Data son utilizadas para analizar a los clientes y sus patrones de consumo y así ofrecer servicios personalizados.

Según la cuarta actualización del Fintech Radar hecha en colaboración con Endeavor México y una encuesta a 148 Startups Fintech, el $45 \%$ de estas empresas usa Big Data y Analítica de Datos, el $43 \%$ utiliza plataformas abiertas y APIs. Además, el $40 \%$ de las Startups Fintech tiene una aplicación móvil y el $26 \%$ utiliza Machine Learning para la implementación de su propuesta de valor.[5]

Los clientes financieros de la Startups Fintech son personas jóvenes en su mayoría con conocimientos tecnológicos y Pequeñas y Medianas Empresas (PyMEs).
Estos representan la fuente principal de ingreso del Ecosistema Fintech.

Por otro lado, las instituciones financieras tradicionales como los bancos, han comenzado a colaborar con las Startups Fintech, evaluando sus modelos de negocios y desarrollando estrategias para adoptar estas nuevas tecnologías financieras, permitiéndoles llegar a nuevos nichos de mercado.

El papel que juega el gobierno es el de proveer una regulación favorable para las Startups Fintech que fomente la innovación y la protección de los inversionistas y consumidores. El gobierno mexicano con la promulgación de la Ley Fintech busca alcanzar cuatro objetivos: uno aumentar la inclusión financiera, dos mejorar la eficiencia del sistema financiero, tres estimular la competencia, y cuatro asegurar la estabilidad financiera.

Por último, se encuentran los Fondos de Capital y Aceleradoras, quienes se encargan de brindar recursos y experiencia a las Startups Fintech. "Los fondos de capital resultan ser el combustible principal que impulsa a que los negocios escalables y con potencial de crecimiento, Ileven a cabo su idea" (BANCOMEXT \& Finnovista, 2018). Los recursos son inyectados a las Startups a través de inversionistas ángel o fondos de capital de riesgo.

Por otra parte las aceleradoras se dedican ha impulsar Startups Fintech que están comenzando a salir al mercado empresarial.

Su funcionamiento se basa en un programa que contiene una serie de convocatorias con un plazo de tiempo estipulado. Durante este programa las aceleradoras ofrecen a las Startups actividades tales como mentoría, formación intensiva, educación digital y tutorización, de manera que obtengan un conocimiento más profundo del ecosistema. (BANCOMEXT \& Finnovista, 2018)

A la fecha se calcula que el agregado de las Startups Fintech mexicanas ha recibido financiamiento por $\$ 800$ millones de USD, dando como promedio $\$ 2$ millones de USD por Startup, esto según Fintech Radar. Asimismo de acuerdo al Fintech Radar cerca del $70 \%$ de las Startups Fintech mexicanas fueron creadas en los últimos 5 años, siendo la ciudad de México el mayor centro de innovación financiera creando el 53\%, siguiéndola Monterrey con el $15 \%$ y Guadalajara con $11 \%$.

\begin{tabular}{|c|c|c|}
\hline Segmentos Fintech & $\begin{array}{c}\text { Numero de } \\
\text { empresas }\end{array}$ & Porcentaje \\
\hline Prestamos & 81 startups & $20.6 \%$ \\
\hline Pagos y Remesas & 79 startups & $20.1 \%$ \\
\hline
\end{tabular}




\begin{tabular}{|c|c|c|}
\hline $\begin{array}{c}\text { Gestión de Finanzas } \\
\text { Empresariales (EFM) }\end{array}$ & 52 startups & $13.2 \%$ \\
\hline $\begin{array}{c}\text { Gestión de Finanzas } \\
\text { Personales (PFM) }\end{array}$ & 39 startups & $9.9 \%$ \\
\hline $\begin{array}{c}\text { Tecnologías } \\
\text { Empresariales para } \\
\text { Instituciones } \\
\text { Financieras (ETFI) }\end{array}$ & 36 startups & $9.1 \%$ \\
\hline $\begin{array}{c}\text { Crowdfunding } \\
\text { Seguros }\end{array}$ & 29 startups & $7.4 \%$ \\
\hline $\begin{array}{c}\text { Puntaje, Identidad y } \\
\text { Fraude }\end{array}$ & 16 startups & $4.1 \%$ \\
\hline Bancos Digitales & 15 startups & $3.8 \%$ \\
\hline $\begin{array}{c}\text { Trading y Mercados } \\
\text { de Capitales }\end{array}$ & 13 startups & $3.3 \%$ \\
\hline $\begin{array}{c}\text { Gestión Patrimonial } \\
\text { (1) }\end{array}$ & 8 startups & $2.0 \%$ \\
\hline
\end{tabular}

Tabla 2. Distribución de las Startups Fintech en México

Fuente: Elaboración propia con información de FINNOVISTA Y ENDEAVOR MÉXICO, "Cuarta actualización del Fintech Radar México", Finnovista, mayo, 2019. Disponible en https://www.finnovista.com/actualizacion-fintech-radarmexico-2019/

El Ecosistema Fintech mexicano tuvo un crecimiento neto del $18 \%$ en el número de Startups existentes desde la última medición hecha por Fintech Radar a finales de julio de 2018. Este crecimiento se dio a pesar de la incertidumbre que genero principalmente a empresas de fondos de pago electrónico y de financiamiento colectivo la promulgación de la Ley Fintech el 9 de marzo de 2018 y posteriormente las primeras disposiciones secundarias el 10 de septiembre de ese mismo año. El Ecosistema Fintech mexicano se posiciono en 2019 como el número uno en América Latina, acercándose a la barrera de las 400 Startups Fintech (394 Startups), esto de acuerdo con Fintech Radar en un informe publicado en noviembre de 2018 por el Banco Interamericano de Desarrollo (BID) y Finnovista.

\section{La Ley Fintech}

La Ley Fintech tiene el objetivo de regular los servicios financieros que ofrecen las Instituciones de Tecnología Financiera (ITF), así como su organización, operación y funcionamiento. También están sujetos a regulación los servicios financieros que sean ofrecidos o realizados por medios innovadores. Esta ley se basa en los principios de inclusión e innovación financiera, promoción de la competencia, protección al consumidor, preservación de la estabilidad financiera, prevención de operaciones ilícitas y neutralidad tecnológica. [6]

La supervisión de la Ley Fintech y las disposiciones están a cargo de la Comisión Nacional Bancaria y de Valores (CNBV) y del Banco de México, así como la Comisión Nacional de Seguros y Fianzas, la Comisión Nacional del Sistema de Ahorro para el Retiro y la Comisión Nacional para la Protección y Defensa de los Usuarios de Servicios Financieros.

La Ley Fintech representa la modernización del sistema financiero mexicano, posicionando a México dentro de las pocas economías que cuentan con una ley de este tipo que regula incluso activos virtuales (criptomonedas) y Modelos Novedosos. "El propósito de la Ley Fintech es dotar de mayor certeza y seguridad jurídica a los inversionistas y usuarios de estas tecnologías, creando para tales efectos a las denominadas Instituciones de Tecnología Financiera (ITF) y sus servicios" (BANCOMEXT \& Finnovista, 2018).

Clasificación de las Instituciones de Tecnología Financiera (ITF):

1. Instituciones de Financiamiento Colectivo (IFC): De acuerdo a la Ley Fintech son todas aquellas actividades destinadas a poner en contacto a personas del público en general, con el fin de que entre ellas se otorguen financiamientos mediante Operaciones realizadas de manera habitual y profesional, a través de aplicaciones informáticas, interfaces, páginas de internet o cualquier otro medio de comunicación electrónica o digital. [4]

Operaciones de las IFC de acuerdo a la Ley Fintech:

- Financiamiento colectivo de deuda, con el fin de que los inversionistas otorguen préstamos, créditos, mutuos o cualquier otro financiamiento causante de un pasivo directo o contingente a los solicitantes.

- Financiamiento colectivo de capital, con el fin de que los inversionistas compren o adquieran títulos representativos del capital social de personas morales que actúen como solicitantes.

- Financiamiento colectivo de copropiedad o regalías, con el fin de que los inversionistas y solicitantes celebren entre ellos asociaciones en participación o cualquier otro tipo de convenio por el cual el inversionista adquiera una parte proporcional o participación en un bien presente 
o futuro o en los ingresos, utilidades, regalías o pérdidas que se obtengan de la realización de una o más actividades o de los proyectos de un solicitante.

2. Instituciones de Fondos de Pago Electrónicos (IFPE): De acuerdo a la Ley Fintech son los servicios realizados con el público de manera habitual y profesional, consistentes en la emisión, administración, redención y transmisión de fondos de pago electrónico, por medio de los actos que a continuación se señalan, a través de aplicaciones informáticas, interfaces, páginas de internet o cualquier otro medio de comunicación electrónica o digital.

Operaciones de las IFPE de acuerdo a la Ley Fintech:

- Abrir y llevar una o más cuentas de fondos de pago electrónico por cada Cliente, en las que se realicen registros de abonos equivalentes a la cantidad de fondos de pago electrónico emitidos contra la recepción de una cantidad de dinero, en moneda nacional o extranjera, o de activos virtuales determinados. Realizar transferencias de fondos de pago electrónico entre sus Clientes mediante los respectivos abonos y cargos en las correspondientes cuentas de fondos de pago electrónico.

- Realizar transferencias de determinadas cantidades de dinero en moneda nacional o, sujeto a la previa autorización del Banco de México, en moneda extranjera o de activos virtuales, mediante los respectivos abonos y cargos en las correspondientes cuentas de fondos de pago electrónico entre sus Clientes y aquellos de otra institución de fondos de pago electrónico, asícomo cuentahabientes o usuarios de otras Entidades Financieras o de entidades extranjeras facultadas para realizar Operaciones de pagos electrónicos.

- Entregar una cantidad de dinero o activos virtuales equivalente a la misma cantidad de fondos de pago electrónico en una cuenta de fondos de pago electrónico, mediante el respectivo cargo en dicha cuenta.

- Mantener actualizado el registro de cuentas de fondos de pago electrónico.

Requisitos generales que deben cumplir las ITF:

- Acta constitutiva debidamente protocolizada por un fedatario público autorizado y en la que se otorguen los poderes suficientes a los representantes legales.
- Estatutos sociales que se ajusten a lo dispuesto por la Ley Fintech.

- Política de divulgación de riesgos y responsabilidades por la realización de las operaciones.

- Medidas y políticas en materia de control de riesgos operativos, así como de seguridad de información, incluyendo las políticas de confidencialidad.

- Plan de negocios.

- Política de prevención de fraude y de operaciones con recursos de procedencia ilícita y financiamiento al terrorismo.

- Información sobre el órgano de administración de la persona moral.

- Procesos de operativos y de control de identificación de sus Clientes.

- Capital mínimo.

\begin{tabular}{|c|c|c|}
\hline & Ventajas & Desventajas \\
\hline $\begin{array}{l}3 . \\
4 . \\
5 .\end{array}$ & $\begin{array}{l}\text { Mitigar el riesgo } \\
\text { de fraudes y } \\
\text { ciberataques. } \\
\text { Mayor } \\
\text { transparencia y } \\
\text { revelación de } \\
\text { información por } \\
\text { parte de la ITF. } \\
\text { Solvencia de las } \\
\text { plataformas. } \\
\text { Prevención de } \\
\text { lavado de dinero. } \\
\text { Mayor } \\
\text { tranquilidad, } \\
\text { seguridad y } \\
\text { confianza por } \\
\text { parte de los } \\
\text { usuarios al } \\
\text { consumir los } \\
\text { productos y } \\
\text { servicios } \\
\text { financieros } \\
\text { ofrecidos por las } \\
\text { ITF. } \\
\text { Mayor inversión } \\
\text { nacional e } \\
\text { internacional al } \\
\text { Ecosistema } \\
\text { Fintech mexicano. } \\
\text { lgualdad de } \\
\text { condiciones para } \\
\text { todas la Startups } \\
\text { Fintech. }\end{array}$ & 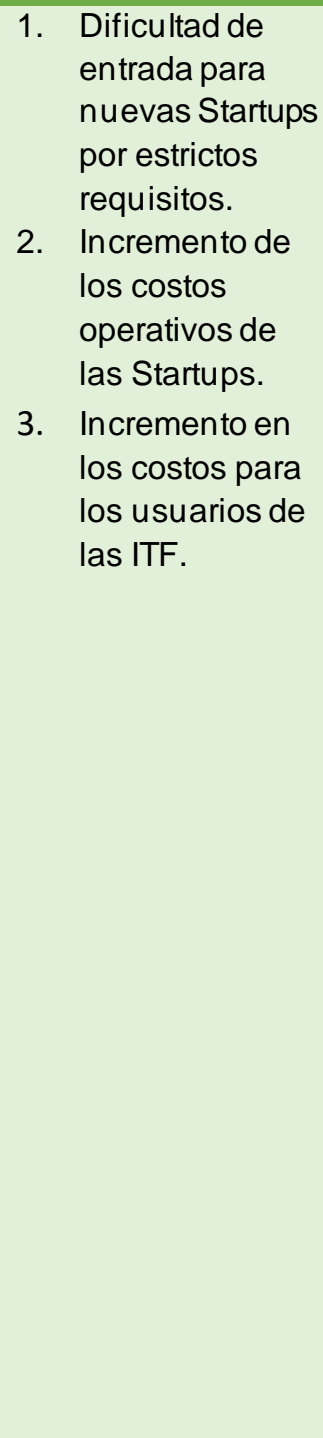 \\
\hline
\end{tabular}


8. Competencia leal.

9. Alianzas

estratégicas con

instituciones del

sector financiero

tradicional y otras

ITF para mejorar

la oferta y

accesos a nuevos

productos y

servicios

financieros.

Tabla 3. Ventajas y desventajas de la Ley Fintech Fuente: Elaboración propia a partir de la información de VIDAL, HUGO, "Las ventajas que ofrecerá la Ley Fintech en el sector financiero, The markethink, marzo, 2018.

\section{Disponible en}

https://www.themarkethink.com/noticias/las-ventajas-queofrecera-la-ley-fintech-en-el-sector-financiero/

La Ley Fintech brinda a las Startups la oportunidad de jugar al mismo nivel de las in stituciones tradicionales, pero para ello se requiere que las nuevas firmas cumplan también con las disposiciones de ley como la de establecer un Gobierno Corporativo.

\section{El impacto de la Ley Fintech y el reto de establecer un Gobierno Corporativo para las Startups}

A pesar del crecimiento de la industria tecnológico financiera en 2018, el sentimiento de las Startups ante la regulación no fue tan bueno. El $53 \%$ de las Startups considero una fuerte barrera de entrada para nuevas Startups la Ley Fintech y $46 \%$ indico que los altos costo asociados al cumplimiento de la Ley generaran ineficiencias, y solo el $24 \%$ de las Startups Fintech estuvieron $100 \%$ conformes con la ley y sus reglamentos, esto de acuerdo a la encuesta hecha por Fintech Radar. Cabe recordar que dichas disposiciones solo son relevantes para entidades de financiamiento colectivo y fondos de pago electrónico.

Directivos de bancos como Citibanamex y BBVA consideran que la regulación permitirá al Ecosistema Fintech consolidarse en el corto plazo, pero solo con las Startups más grandes. Juan Guerra director ejecutivo de Innovación de Citibanamex en una entrevista publicada en el Economista comento que la regulación impone criterios en la industria que hace para algunos sectores no sea rentable o costeable seguir participando. [7]

Entre las disposiciones que establece la Ley Fintechy que representan un reto para las nuevas empresas de tecnología financiera se encuentra el establecimiento de un Gobierno Corporativo. Estas disposiciones en materia de seguridad y transparencia tienen el objetivo de preservar la estabilidad y correcto funcionamiento de las Instituciones de Tecnología Financiera (ITF), abarcando temas de control interno y administración de riesgos.

Para poder realizar sus operaciones, ofrecer sus servicios, prevenir conflictos de intereses e identificar a sus clientes, las empresas de tecnología financiera deberán sujetarse a la disposición de establecer un Gobierno Corporativo y además a una regulación estricta de sanas prácticas de auditoria, de contabilidad, de revelación de información, de transparencia y de equidad en las actividades y servicios que ofrecen.

El Gobierno Corporativo es el marco de normas y prácticas, que se refieren a las estructuras y procesos para la dirección de una empresa, por el cual un consejo de administración asegura la rendición de cuentas, la equidad y la transparencia en la relación de la empresa con todas sus partes interesadas (la junta directiva, los accionistas, clientes, empleados, gobierno y la comunidad). (Bolsa Mexicana de Valores, 2015) [8]

El gobierno corporativo se basa en principios como la equidad, la honestidad, la solidaridad y la justicia, para proteger los intereses de sus inversionistas, clientes y todos los demás grupos de interés, promoviendo la transparencia, la productividad y la integridad de las instituciones.

\begin{tabular}{|l|}
\hline Beneficios del Gobierno Corporativo \\
1. El trato igualitario y la protección de los \\
intereses de todos los accionistas. \\
2. El reconocimiento de los terceros interesados \\
en el buen desempeño, la estabilidad y la \\
permanencia en el tiempo de la sociedad. \\
3. La emisión y revelación responsable de la \\
información, así como la transparencia en la \\
administración. \\
4. Asegurar que exista la visión estratégica de \\
la sociedad, así como la vigilancia y el \\
efectivo desempeño de la administración. \\
5. El ejercicio de la responsabilidad fiduciaria \\
del Consejo de Administración. \\
6. La identificación, administración, control y \\
revelación de los riesgos a que está sujeta la \\
sociedad. \\
7. La declaración de principios éticos y de \\
responsabilidad social empresarial. \\
8. La prevención de operaciones ilícitas y \\
conflictos de interés. \\
9. La revelación de hechos indebidos y la \\
protección a los informantes.
\end{tabular}


10. El cumplimiento de las distintas regulaciones a que esté sujeta la sociedad.

11. El dar certidumbre y confianza a los inversionistas y terceros interesados sobre la conducción honesta y responsable de los negocios de la sociedad.

Tabla 4. Beneficios del gobierno corporativo Fuente: Elaboración propia con base en RUIIZ, JOAQUÍN Y NAVARRO, JUAN, “Gobierno Corporativo", Ejecutivos de Finanza. Comité Técnico Local (IMEF), 2011. Disponible en

https://www.imef.org.mx/grupos/guadalajara/descargas/g obcorporativo/GobCorporativo1.pdf

Estructura del gobierno corporativo.

El Gobierno Corporativo está conformado por un Consejo de Administración y comités de prácticas societarias, incluyendo un Comité de Auditoria.

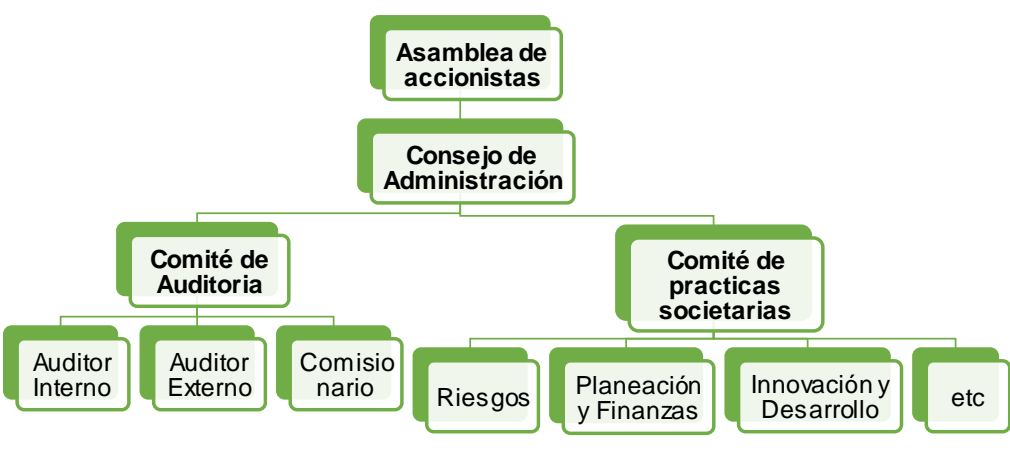

Figura 1. Estructura del gobierno corporativo Fuente:Adaptación de Asesores de Consejo y Alta Dirección S.C/Board Solutions LLC. Disponible en https://www.bmv.com.mx/es/mi-empresa-enbolsa/gobierno-corporativo

Las acciones que deben realizarse para establecer un Gobierno Corporativo se dividen en dos etapas de acuerdo con el Grupo Bolsa Mexicana de Valores:

1. Primera Etapa de Institucionalización:

- Implementar un Consejo de Administración y Comités.

- Operar conforme a la LGSM y al Código de Mejores Prácticas Corporativas.

- Incorporación de consejeros independientes en el Consejo y para presidir el Comité de apoyo.

\section{Segunda Etapa de Institucionalización:}

- Modelo de Gobierno Corporativo Óptimo:

- Operando conforme a las prácticas internacionales de Gobiemo Corporativo.

- Operación con consejeros independientes en Comités.

- Separación de Comités.

Pero a pesar de los múltiples beneficios que brinda la implementación de un Gobierno Corporativo a las empresas, su establecimiento plantea algunos desafíos provocando que la mayoría las Startups comiencen sus operaciones sin dicho Gobierno Corporativo. "Por lo general el emprendedor líder y du eño de la empresa es quien ejerce el rol de gerente y toma la mayoría de las decisiones que afectan a la empresa, por no decir que todas" (Ruiz G \& Toro V, 2016). [9]

Lo anterior se debe a que todas la Startups comparten una serie de características que constituyen su naturaleza. Algunas de estas características son que no existe jerarquía, todos los miembros de la empresa siguen un mismo objetivo, los trabajadores hacen lo que más les gusta ocupando su puesto ideal, existe fuerte liderazgo por parte del dueño o fundador de la empresa, la satisfacción al cliente es lo más importe y por último el ambiente laboral es agradable.

En sus inicios la mayoría de las Startups no cuenta con políticas en materia de control de riesgos operativos, de seguridad de la información, de confidencialidad, de prevención de fraudes, y de operaciones con recursos de procedencia ilícita y financiamiento al terrorismo, y para que establezcan un Gobierno Corporativo se necesita un tiempo considerable. El problema para las Startups Fintech en México es que el $35 \%$ de estas empresas tienen menos de 3 años operando, el $20 \%$ tiene de 3 a 4 años, el $17 \%$ tiene de 4 a 5 años y solo el $28 \%$ tiene más de 5 años en operación de acuerdo a Fintech Radar.

En México con la Ley de Mercado de Valores comenzó a trascender el desarrollo del Gobierno Corporativo mediante la aplicación de Mejores Prácticas Corporativas, código emitido en 2006 por la Comisión Nacional Bancaria y de Valores (CNBV). Pero muchos empresarios y directivos consideran un gasto innecesario y un riesgo dar a conocer informacion de la empresa. También consideran que la implementación de un Gobierno Corporativo significaba la pérdida del control por parte de los accionistas o dueños de la empresa.

Actualmente las Startups se encuentran con la necesidad de establecer un Gobierno Corporativo cuando los fondos de capital de riesgo les exigen un asiento en el consejo como parte del trato de financiamiento. Así como también 
cuando se enfrentan a un proceso de valoración, la falta de mecanismos de un Gobierno Corporativo influye en el valor de la empresa. Si las Startups Fintech contaran con un mecanismo de Gobierno Corporativo que disminuya riesgos, les permitiría acceder a mejores fuentes de financiamiento y captar mayor inversión. [10]

\section{Conclusión}

El resultado de la regulación para empresa de tecnología financiera en México es un ecosistema Fintech más consolidado y atractivo para inversionistas nacionales y extranjeros. Las Startups Fintech que logren la autorización de la Comisión Nacional Bancaria y de Valores (CNBV) para operar de forma legal podrán atender a mayor número de inversionistas, debido a que contaran con un marco regulatorio especifico que dará certeza a los inversores. Anteriormente la mayor parte de la inversión a estas empresas provenía de personas físicas, pero con la Ley Fintech, fondos de inversión y bancos están comenzando a invertir en las Startups Fintech.

Fintech Radar identifico 394 Startups Fintech en México de las cuales solo 200 empresas, que representan el $50.76 \%$ de la industria, encajaron en la regulación. Al vencimiento del plazo para entregar solicitud ante la Comisiona Nacional Bancaria y de Valores (CNBV) para operar de manera formal, solo 85 Startups Fintech registraron su solicitud, lo que significa que del total de la industria Fintech en México solo el $21.57 \%$ podrá continuar con sus operaciones de forma legal. De las 85 empresas que registraron su solicitud, 60 solicitaron autorización para operar como institu ciones de fondos de pago electrónico, y 25 como instituciones de financiamiento colectivo.

Algunas de las Fintech que sí presentaron su solicitud de autorización formal ante la CNBV son: Doopla, Yotepresto, Prestadero, Zigo Capital y Expansive." Pero aquellas fintechs que no presentaron su solicitud de autorización "deberán abstenerse" de seguir operando y deberán realizar únicamente los actos destinados a la conclusión o cesión de las operaciones existentes" (Saldívar, 2019). [11]

Algunas Startups decidieron no entregar solicitud para operar de forma legal como Fintech ante la Comisión Nacional Bancaria y de Valores (CNBV) debido a que consideraron los requisitos demasiado restrictivos y no pudieron acatarse a ellos. Empresas como Doopla empresa mexicana de Crowdfunding, menciona que fue un arduo trabajo poder atender los requisitos y en tregar la solicitud ante la Comisión Nacional Bancaria y de Valores (CNBV), dedicando más de ocho meses y 3500 horashombre y más de 30 documentos conformados.
Doopla reconoce que ser una empresa con más de cuatro años operando, les dio gran ventaja, pero considera que para empresas más jóvenes los requisitos representan medidas muy rigurosas que deben cumplir principalmente en temas de gobierno corporativo, preven ción de lavado de dinero y financiamiento al terrorismo y ciberseguridad. Ahora que Doopla haingresado su solicitud formal ante la Comisión Nacional Bancaria y de Valores (CNBV) deberá esperar una respuesta que será emitida a principios de 2020, la cual el directivo espera sea positiva.

Por otra parte tal y como dijo Simon Dalgleish, director de Operaciones de M2Crowd, empresa de fondeo colectivo: "es un hecho que el mercado se reducirá, pero la industria de la tecnología financiera requiere de cierto nivel de profesionalismo y certidumbre para poder crecer y al irse algunas empresas ayudará a fortalecer la industria en general" (Gutiérrez, 2019). [12]

La Ley Fintech fortalecerá la industria de la tecnología financiera en México. Pero en el corto plazo solo las Startups Fintech más grandes y con más tiempo en operación serán las que consolidaran el Ecosistema Fintech. Los requisitos de esta ley como el de establecer mecanismos de Gobierno Corporativo representan un reto para aquellas Startups Fintech que tienen menos de cuatro años operando, las cuales conforman el $55 \%$ de la industria Fintech. Dado lo anterior el crecimiento de la industria de la tecnología financiera en México podría disminuir a comparación del crecimiento visto antes de la Ley Fintech.

\section{Referencias}

[1] BID, BID Invest, \& Finnovista. (2018). Crecimiento y Consolidación. BID, 2, 28-32. https://www.finnovista.com/informe-fintech-2018/

[2] Condusef (2018). El ABC de la Ley Fintech. Proteja su dinero, 21. https://www.condusef.gob.mx/Revista/PDF-s/2018/224/fin.pdf

[3] López, M., \& González, M. (2018). Ley Fintech: Regulación vs Innovación y Competenciá. Sánchez Ugarrete y Aznar, 1, 3. https://amexcap.com/contenido/ley-fintech-regulacion-vs-innovaci-ny-competencia-ksa

[4] BANCOMEXT, \& Finnovista.(2018). FINTECH EN EL MUNDO. La revolución digital de las finanzas ha llegado a México: Descripción de ecosistema. Coordinanción Editorial Académica Universidad Anáhuac México, 1, 16-17. https://www.bancomext.com/wpcontent/uploads/2018/11/Libro-Fintech.pdf

[5] Finnovista, \& Endeavor México. (2019). El ecosistema Fintech mexicano recupera el liderazgo en América Latina y se acerca a la barrera de las 400 startups. Finnovista, 4. https://www.finnovista.com/actualiza cion-fintech-ra dar-mexico-2019/

[6] SHCP, (2018). Ley para regular las Intiticiones de Tecnología Financiera.

http://www.diputados.gob.mx/LeyesBiblio/pdf/LRITF_090318.pdf

[7] Juárez, E. (2019). Bancos ven consolidación del sector fintech; quedarán los más grandes. El Economista. https://www.eleconomista.com.mx/sectorfinanciero/Bancos-venconsolidacion-del-sector-fintech-quedaran-los-mas-grandes-201909160052.html 
[8] Bolsa Mexicana de Valores. (2015). Gobierno Corporativo. https://www.bmv.com.mx/es/mi-empresa-en-bolsa/gobiernocorporativo.

[9] Ruiz G, \& Toro V. (2016). Valoración de una start-up de tecnología. Universidad EAFIT, 14. https://core.ac.uk/download/pdf/47252906.pdf

[10] Expansión. (2018). OPINIÓN: La importancia de un buen gobierno corporativo en las 'startups'. Expansión. https://expansion.mx/opinion/2018/03/16/opinion-la-importancia-deun-buen-gobierno-corporativo-en-las-startups

[9] Saldívar, B. (2019). "El futuro nos alcanzó”, resume la CNBV respecto a las $\quad$ Fintech. El Economista. https://www.eleconomista.com.mx/sectorfinanciero/El-futuro-nosalcanzo-resume-la-CNBV-respecto-a-las-Fintech-20190927-0081.html

[10] Gutiérrez, F. (2019). Ecosistema fintech se vuelve más atractivo. El Economista.

https://www.eleconomista.com.mx/sectorfinanciero/Ecosistema-

fintech-se-vuelve-mas-atractivo-20190925-0107.html 